\title{
Research Report About IOT Security
}

\author{
Christos Beretas* \\ PhD Candidate in Cyber Security at Innovative Knowledge Institute, Paris, France. \\ *Corresponding Author: Christos Beretas, PhD Candidate in Cyber Security at Innovative Knowledge
} Institute, Paris, France

\begin{abstract}
The purpose of the present research is to identify security holes in IoT in percentage, according to a research conducted from January 5, 2020 to January 26, 2020 involving a large number of people who are actively involving with IoT. These people wanted to share their fears and anxieties, expressing their views freely. The majority of participants expressed concerns about IoT security. This research clearly shows the phobias that exist as well as the lack of an IoT protection mechanism considering the restrictions that exist.
\end{abstract}

\section{INTRODUCTION}

There is a significant increase in the use of IoT in various sectors, the use of IoT has increased significantly in the last 5 years, taking into account the technological innovation of both electronics and Information Technology. According to research published in recent years, the use of IoT in the next decade is expected to increase by as much as $90 \%$ as human needs grow and technology becomes more accessible.

Given recent forecasts for the use of IoT in the future, what concerns tech-savers is the lack of a security strategy that will be able to prevent IoT from being attacked by external cyber-attacks or cyber-attacks that began from the internal network. Some research that have been performed with a variety of prosthetic techniques have shown to be insufficient and do not provide the protection that is required.

In order to create this research report, all the parameters were taken into account, including the required evaluations, then the questionnaire sent to the participants after considering the possibility of misinterpretation.

\section{Method}

For the purpose of this research, one electronic questionnaire was used which was distributed to groups on social media. Participation was anonymous and voluntary. There was no personal data collection, the duration of this survey was January 5, 2020 to January 26, 2020 with a total duration of 22 days.

\section{RESUlts}

The participation rate in the questionnaire was $89 \%$. The following table presents in detail the research data which clearly shows that the issue of IoT security and privacy is a burning issue that will be of concern to all involved. In one or the other way with IoT. From the analysis of the above table, may conclude the participants\&\#39; clear concern about the security of Iot and its immediate improvement at $87 \%$.

\section{ANALYSIS}

In $87 \%$ of survey participants are deeply concerned about the security of IoT and requesting for its immediate improvement. This is followed by $76 \%$ of those who believe that improvements and enhancements to security levels are needed.

An $11 \%$ believe that a breach of an IoT cannot affect the rest of the network. 9\% believe that deploying IoT in lower importance infrastructures would solve the security problem, thinking that breaching low importance information systems would not significantly affect organic information technology infrastructures. $7 \%$ believe that IoT is secure, and finally $30 \%$ believe there are security holes but they are not sure. 


\section{CONCLUSION}

This research report has clearly raised users\&\#39; concerns in the field of IoT security. It has highlighted the need to immediately improve data security and network security policy. The results showed some users in very small numbers who were not concerned about IoT security. The vast majority accept that there are problems and worries about personal data, and acknowledges the need to immediately improve and enhance IoT security

\section{REFERENCES}

[1] Christos Beretas, MSc Researcher in Cyber Security at Global Foundation for Cyber Studies and Research. Washington D.C, USA.

[2] Cyber Defence $/ \mathrm{PhD}$ Candidate in Cyber Security (Innovative Knowledge Institute) Paris, France

[3] Christos Beretas on top 100 cyber security experts in the world Information Technology / Security Specialist Fortinet Network Security Expert Certificates (NSE1/NSE2/NSE3)

[4] Member of Alpha Beta Kappa Honor Society, Alpha of Ohio, USA Academia: https://independent.academia.eu/ Christos Beretas Web: https://www.christosberetas.com

Citation: Christos Beretas, "Research Report About IOT Security." International Journal of Innovative Research in Electronics and Communications (IJIREC), 7(1), pp.8-9. http://dx.doi.org /10.20431/23494050.0701002

Copyright: () 2020 Authors this is an open-access article distributed under the terms of the Creative Commons Attribution License, which permits unrestricted use, distribution, and reproduction in any medium, provided the original author and source are credited. 